\title{
Ocriplasmin in the Treatment of Vitreomacular Traction in a Patient with Central Retinal Vein Occlusion: A Case Report
}

\author{
Alessandra Rosati Raffaele Antonio Esposito Giuseppe Mannino \\ Gianluca Scuderi \\ Ophthalmology Unit, NESMOS Department, S. Andrea Hospital, Faculty of Medicine and \\ Psychology, University of Rome "La Sapienza", Rome, Italy
}

\section{Keywords}

Posterior vitreous detachment · Vitreomacular adhesion · Vitreomacular traction · Ocriplasmin

\begin{abstract}
Aim: To investigate the efficacy of intravitreal injection of ocriplasmin (JETREA ${ }^{\circledR}$ ) in the treatment of vitreomacular traction (VMT). Materials and Methods: An 81-year-old man with VMT associated with central retinal vein occlusion in his left eye, was treated with a single intravitreal injection of ocriplasmin $(25 \mu \mathrm{g})$. Best corrected visual acuity (BCVA), ocular fundus, and optical coherence tomography were examined before and after treatment. Results: Complete release of VMT produced a reduction of central macular thickness, ranging from 459 to $141 \mu \mathrm{m}$. BCVA remained stable. Discussion and Conclusions: The use of ocriplasmin was effective in the treatment of VMT. Ocriplasmin represents a valid alternative to conventional pars plana vitrectomy.

\section{Introduction}

Vitreomacular traction (VMT) is a complication of posterior vitreous detachment (PVD) characterized by distortion of the retinal profile, resulting in metamorphopsia and visual loss. 
The vitreous gel undergoes degenerative processes such as synchysis (liquefaction and formation of so-called lacunae) and syneresis (total collapse of the collagen fibers) which, together with the reduction of its water content, play an important role in determining the detachment of the posterior hyaloid from the retinal surface. Adapted from Uchino et al. [1] and Johnson [2], four stages of PVD have been identified. Focal perifoveal PVD occurs in around $50 \%$ at ages $30-39$ years, whereas complete PVD is observed in 50\% or more of individuals aged 70 years or older [3]. The main causes include: trauma, refractive errors (particularly myopia), surgery, inflammatory processes, and decreased estrogen levels in postmenopausal women. The process is usually asymptomatic; in a few cases, it may be complicated by vitreomacular adhesion (VMA) between the vitreous cortex and the macular area. VMA is the persistent attachment of the posterior hyaloid to the macula during the formation of a partial PVD. It may produce tractional forces that lead to a spectrum of disorders, such as VMT syndrome and full-thickness macular hole [4]. Various factors may affect the development or severity of the disease: vitreous liquefaction and anatomical focal factors that may increase foveal adhesion and integrity of internal limiting membrane (ILM). Therefore, VMT has been associated with other conditions such as exudative age-related macular degeneration (ARMD), diabetic macular edema (DME), retinal vein occlusion (RVO), and epiretinal membrane (ERM). It may exacerbate cystoid macular edema associated with ERM, RVO, and diabetic retinopathy [5, 6]. Simple (noncontractile) ERM is associated with retinal surface wrinkling or "cellophane" maculopathy. However, the contraction of myofibroblasts within the more complex type of ERM has been proposed to exert a tangential traction at the vitreoretinal interface (VRI), which can result in retinal puckering, thickening, folding, or detachment, together with vascular distortion. An enzyme which acts against molecular substrates responsible for VMA has been successfully used to induce vitreolysis. Plasmin and a truncated form of plasmin, ocriplasmin (formerly microplasmin), have been shown to be effective in ex vivo studies of vitreolysis both in animals and in humans. Ocriplasmin is a recombinant serine protease with activity against fibronectin and laminin, components of the VRI. Clinical studies suggested the role in inducing liquefaction of the vitreous and the consequent separation from the retina. For this purpose, an intravitreal injection of ocriplasmin (JETREA ${ }^{\circledR}$ ), as nonsurgical treatment of VMT, was performed. The advent of optical coherence tomography (OCT) has proved a great benefit to the study of anatomy and disorders of the VRI. Spectral domain (SD)-OCT or time domain-OCT have similar ability to assess VRI abnormalities and outcomes of enzymatic vitreolysis. SD-OCT may be superior to formal clinical trial grading due to greater interreader reproducibility and, therefore, decreased need for arbitration of discrepant values [7].

\section{Case Report}

An 81-year-old man, affected by arterial hypertension treated with amlodipine and acetylsalicylic acid, presented in May 2014 with a strong visual loss due to a central RVO (CRVO) in his left eye that occurred in 2011 (Fig. 1) and for which he had undergone two intravitreal Ozurdex injections in January and October 2013. His medical history revealed a previous CRVO also in his right eye, but it was ignored and never subjected to treatment. At the time of first examination, his uncorrected visual acuity was hand motion in his right eye and his best corrected visual acuity (BCVA) 1 in his left eye. Intraocular pressure was $10 \mathrm{~mm} \mathrm{Hg}$ in his right eye and $16 \mathrm{~mm} \mathrm{Hg}$ in the left one. Slit lamp examination showed bilateral pseudophakia; dilated fundus examination presented bilateral outcomes of CRVO. SD-OCT identified a notable 
VMT in the left eye characterized by an alteration of the macular profile and an increase of central retinal thickness $(459 \mu \mathrm{m})$ with multiple intraretinal pseudocysts. Interruption of photoreceptor inner segment/outer segment (IS/OS) junctional layer and altered reflectivity and morphology of the retinal pigmented epithelium/choriocapillary complex were also evident (Fig. 2). In June 2014, the patient received a single 125- $\mu$ g ocriplasmin intravitreal injection in his left eye. Two months later, BCVA was stable, anterior segment was quiet, and dilated fundus examination showed a complete release of VMT. Specifically, OCT scans demonstrated the complete release of VMT, characterized by an improvement of the retinal profile with reappearance of the foveal pit and a significant central macular thickness reduction $(141 \mu \mathrm{m})$. Slightly increased paracentral macular thickness persisted, especially nasal to the fovea (285 $\mu \mathrm{m})$, although it was remarkably reduced compared to the previous examination. It finally showed a slight irregularity of the ILM with small intraretinal pseudocystic spaces and persistent deconstruction of subfoveal IS/OS junctional layer (Fig. 3). In December 2014, the left eye remained stable, as revealed by an OCT scan (Fig. 4).

\section{Discussion and Conclusions}

Ocriplasmin is a promising recombinant protease with activity against fibronectin and laminin, components of the vitreoretinal interface. Recently, prospective phase III trials have shown their efficacy and safety in the treatment of symptomatic VMA. Particularly, the MIVITRUST program comprised 2 large multicenter, prospective, randomized, placebo-controlled, phase III trials (MIVI-006 and MIVI-007) that compared the efficacy of a single 125- $\mu \mathrm{g}$ ocriplasmin intravitreal injection (JETREA ${ }^{\circledR}$ ) with a single placebo injection for obtaining VMA release without vitrectomy [8]. A variety of potential vitreolytic agents have been tested, including collagenase, chondroitinase, hyaluronidase, dispase, nattokinase, plasmin, plasminogen activators (tPA and urokinase) and microplasmin $[9,10]$. Nevertheless, most of the agents examined to date have been abandoned because of toxicity, insufficient clinical efficacy, or both. Based on the promising profile reported, ocriplasmin is nowadays the only molecule employed. Our case report revealed the importance of the results regarding the anatomic aspect of VMT. The complete release of VMT allowed to achieve a relevant morphological improvement of the macular area with a decrease in central macular thickness. However, visual loss remained stable because of the irreversible degenerative changes of subfoveal IS/OS junctional layer, evaluated by OCT. The improvement in visual acuity is variable and only depends on retinal layer integrity. In a prospective trial, where 8 eyes were included using a single administration of intravitreal ocriplasmin, $62.5 \%$ of patients ( $5 / 8$ eyes) experienced complete release of the VMT. Of the 5 patients with complete release of VMT, 3 patients had a one-line worsening of their vision, 1 had a 4 -line improvement of vision, and 1 stayed the same. There were no complications [11]. Stalmans et al. [8] initially reported a dose- and time-dependent cleavage between the posterior hyaloid and the ILM created by ocriplasmin without any adverse effects on retinal structure in both human cadaver and feline eyes. Doses $\geq 125.0 \mu \mathrm{g}$ (equivalent to 2 units of plasmin) produced a complete PVD with bare ILM in the human eyes, as demonstrated by electron microscopy. According to MIVI-TRUST phase III clinical trial data, most of ocular adverse events happened during the first week after injection, and the most common ones were floaters, ocular pain, visual loss, and phosphenes [8]. However, they were less than the risks related to pars plana vitrectomy (PPV), such as infection, bleeding, retinal detachment, increase in intraocular pressure, and cataract [12]. Basically, there are three alternative treatments for the resolution of a VMT causing a significant visual loss: 


\section{Case Reports in Ophthalmology}

\begin{tabular}{l|l}
\hline DOI: $10.1159 / 000491487$ & (c) 2018 The Author(s). Published by S. Karger AG, Basel \\
\hline
\end{tabular} www.karger.com/cop

Rosati et al: Ocriplasmin in the Treatment of Vitreomacular Traction in a Patient with Central Retinal Vein Occlusion: A Case Report

vitrectomy, pneumatic vitreolysis, and ocriplasmin. Regarding the efficacy and safety profile, ocriplasmin is more advantageous than intravitreal injection of perfluoropropane (C3F8) (pneumatic vitreolysis) and vitreal surgery, without the concern for the high rate of cataract formation seen with PPV [13]. There are currently no published consensus guidelines for the management of VMT. However, some general recommendations can be made. Patients should be observed for 2-3 months to see if spontaneous resolution occurs, although this is dependent on symptom severity and impact on the individual patient's quality of life. If there is no resolution, treatment with ocriplasmin should be considered, taking into account factors such as the width of the VMA, presence of ERM, the status of the affected eye's lens, and patient age. Surgery, as opposed to ocriplasmin, is recommended if significant ERM is present [3]. Positive results from trials have made ocriplasmin a promising pharmacologic agent for the treatment of symptomatic VMA. Ongoing studies and investigations that should be launched soon will certainly help improve those results by specifying the indications, and perhaps broadening them as well. Considering its ability to induce PVD, other ophthalmic conditions and pathologies related to VMA might benefit from ocriplasmin intravitreal injection, i.e. DME, ARMD, vitreoretinal surgery, uveitic macular edema, pediatric eyes before vitrectomy; nevertheless, first results were not as satisfying as the ones achieved in the treatment of VMA [14, 15]. Further research is needed to better understand the clinical indications, visual improvement and, most of all, potential interactions with other medications.

\section{Statement of Ethics}

The authors have no ethical conflicts to declare.

\section{Disclosure Statement}

The authors have no conflicts of interest to declare.

\section{References}

1 Uchino E, Uemura A, Ohba N. Initial stages of posterior vitreous detachment in healthy eyes of older persons evaluated by optical coherence tomography. Arch Ophthalmol. 2001 Oct;119(10):1475-9.

2 Johnson MW. Posterior vitreous detachment: evolution and complications of its early stages. Am J Ophthalmol. 2010 Mar;149(3):371-82.e1.

3 Steel DH, Lotery AJ. Idiopathic vitreomacular traction and macular hole: a comprehensive review of pathophysiology, diagnosis, and treatment. Eye (Lond). 2013 Oct;27(S1 Suppl 1):S1-21.

4 Duker JS, Kaiser PK, Binder S, de Smet MD, Gaudric A, Reichel E, et al. The International Vitreomacular Traction Study Group classification of vitreomacular adhesion, traction, and macular hole. Ophthalmology. 2013 Dec;120(12):2611-9.

5 Jackson TL, Nicod E, Angelis A, Grimaccia F, Prevost AT, Simpson AR, et al. Vitreous attachment in agerelated macular degeneration, diabetic macular edema, and retinal vein occlusion: a systematic review and metaanalysis. Retina. 2013 Jun;33(6):1099-108.

6 Koizumi H, Spaide RF, Fisher YL, Freund KB, Klancnik JM Jr, Yannuzzi LA. Three-dimensional evaluation of vitreomacular traction and epiretinal membrane using spectral-domain optical coherence tomography. Am J Ophthalmol. 2008 Mar;145(3):509-17.

7 Folgar FA, Toth CA, DeCroos FC, Girach A, Pakola S, Jaffe GJ. Assessment of retinal morphology with spectral and time domain OCT in the phase III trials of enzymatic vitreolysis. Invest Ophthalmol Vis Sci. 2012 Oct;53(11):7395-401. 


\section{Case Reports in Ophthalmology} \begin{tabular}{l|l}
\hline DOI: 10.1159/000491487 & $\begin{array}{l}\text { ○ } 2018 \text { The Author(s). Published by S. Karger AG, Basel } \\
\text { www.karger.com/cop }\end{array}$
\end{tabular}

Rosati et al: Ocriplasmin in the Treatment of Vitreomacular Traction in a Patient with Central Retinal Vein Occlusion: A Case Report

8 Stalmans P, Benz MS, Gandorfer A, Kampik A, Girach A, Pakola S, et al.; MIVI-TRUST Study Group. Enzymatic vitreolysis with ocriplasmin for vitreomacular traction and macular holes. N Engl J Med. 2012 Aug;367(7):606-15.

9 Schneider EW, Johnson MW. Emerging nonsurgical methods for the treatment of vitreomacular adhesion: a review. Clin Ophthalmol. 2011;5:1151-65.

10 Johnson MW, Brucker AJ, Chang S, Gandorfer A, Kampik A, Kuppermann BD, et al. Vitreomacular disorders: pathogenesis and treatment. Retina. 2012 Sep;32 Suppl 2:S173.

11 Knudsen VM, Kozak I. A retrospective study of a single practice use of ocriplasmin in the treatment of vitreomacular traction. Saudi J Ophthalmol. 2014 Apr;28(2):139-44.

12 Jackson TL, Nicod E, Angelis A, Grimaccia F, Prevost AT, Simpson AR, et al. Pars plana vitrectomy for vitreomacular traction syndrome: a systematic review and metaanalysis of safety and efficacy. Retina. 2013 Nov-Dec;33(10):2012-7.

13 Johnson MW. How should we release vitreomacular traction: surgically, pharmacologically, or pneumatically? Am J Ophthalmol. 2013 Feb;155(2):203-205.e1.

14 Stefanini FR, Maia M, Falabella P, Pfister M, Niemeyer M, Kashani AH, et al. Profile of ocriplasmin and its potential in the treatment of vitreomacular adhesion. Clin Ophthalmol. 2014 May;8:847-56.

15 Song SJ, Smiddy WE. Ocriplasmin for symptomatic vitreomacular adhesion: an evidence-based review of its potential. Core Evid. 2014 Mar;9:51-9.

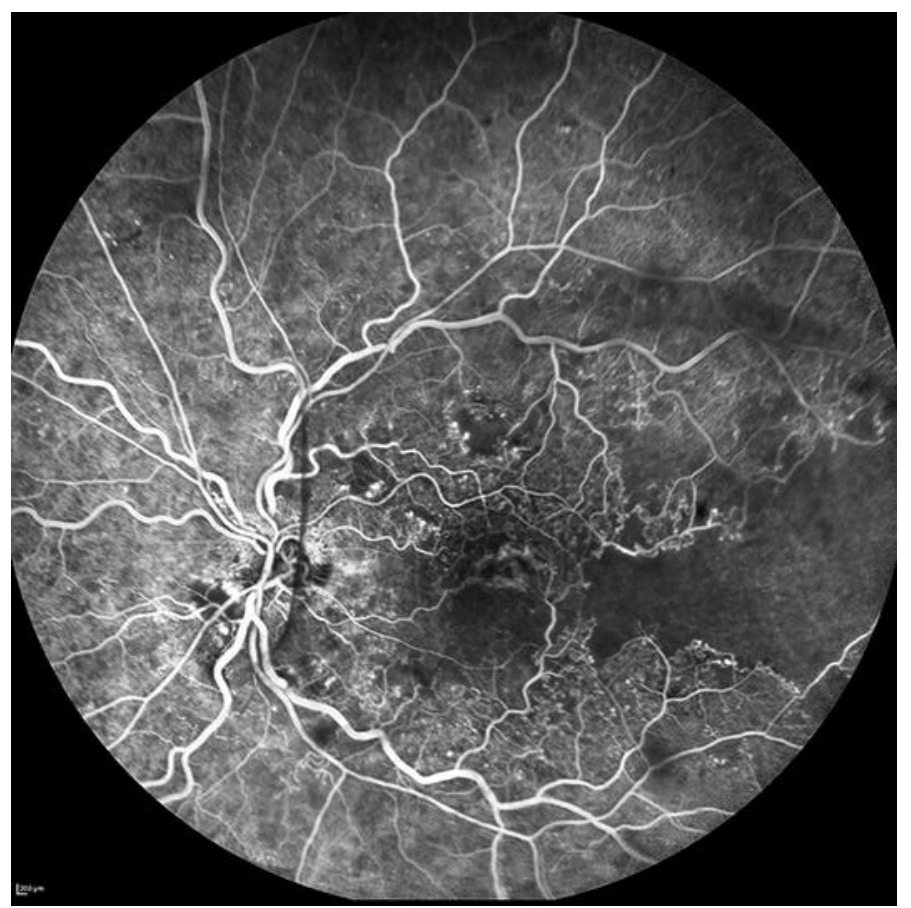

Fig. 1. Fluorescein angiography of the left eye with central retinal vein occlusion showing marked hypofluorescence secondary to widespread capillary nonperfusion. 


\section{Case Reports in Ophthalmology}

\begin{tabular}{l|l}
\hline Case Rep Ophthalmol 2018;9:357-364 \\
\hline DOI: 10.1159/000491487 & $\begin{array}{l}\text { @ 2018 The Author(s). Published by S. Karger AG, Basel } \\
\text { www.karger.com/cop }\end{array}$ \\
\hline
\end{tabular}

Rosati et al: Ocriplasmin in the Treatment of Vitreomacular Traction in a Patient with Central Retinal Vein Occlusion: A Case Report
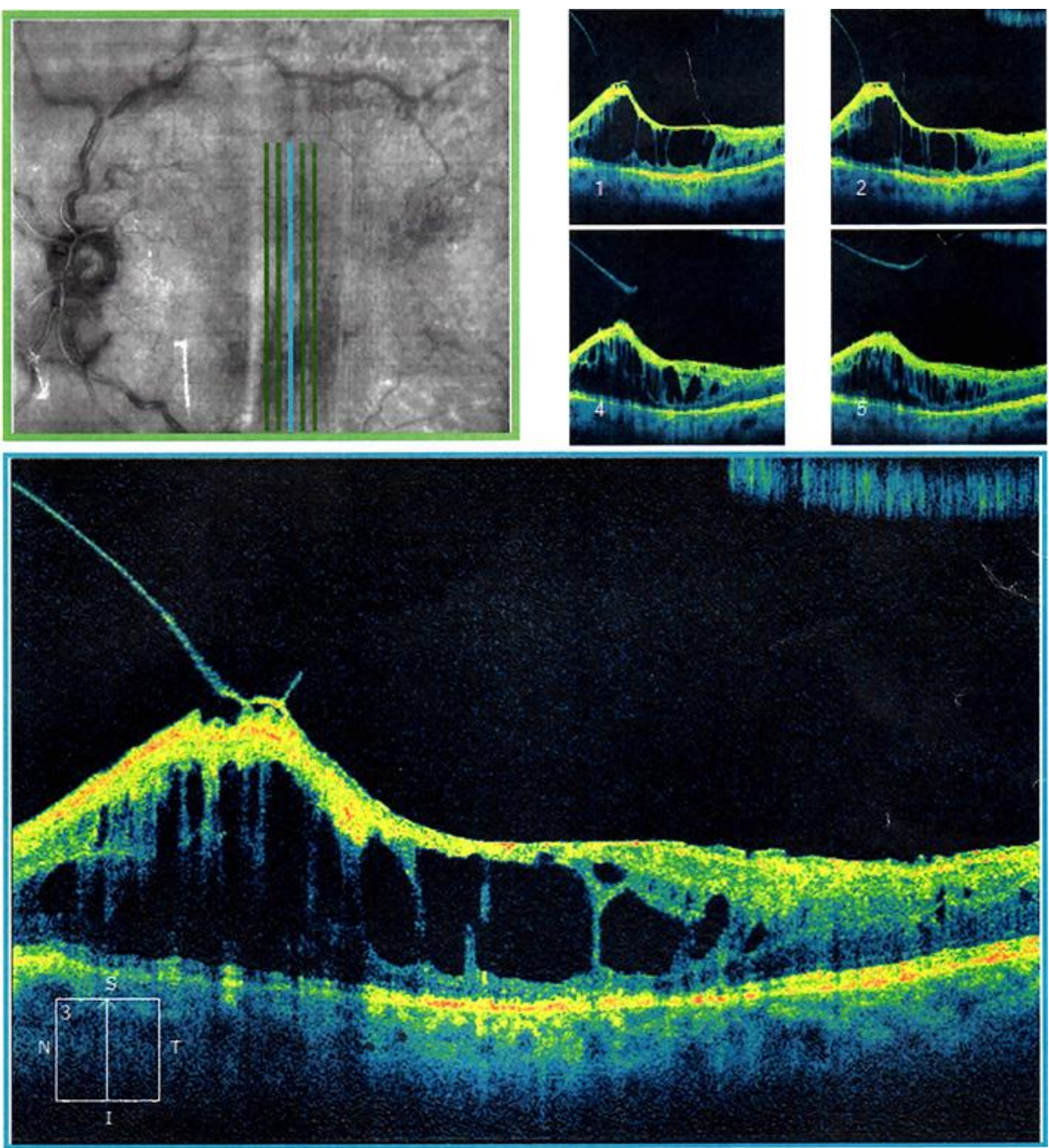

Fig. 2. Left eye SD-OCT performed before ocriplasmin intravitreal injection revealing a remarkable VMT characterized by an alteration of the macular profile and an increase in central retinal thickness $(459 \mu \mathrm{m})$ with multiple intraretinal pseudocysts. Interruption of photoreceptor IS/OS junctional layer and altered reflectivity and morphology of the retinal pigmented epithelium/choriocapillary complex were also evident. 


\section{Case Reports in Ophthalmology}

\begin{tabular}{l|l}
\hline Case Rep Ophthalmol 2018;9:357-364 \\
\hline DOI: 10.1159/000491487 & $\begin{array}{l}\text { @ 2018 The Author(s). Published by S. Karger AG, Basel } \\
\text { www.karger.com/cop }\end{array}$ \\
\hline
\end{tabular}

Rosati et al: Ocriplasmin in the Treatment of Vitreomacular Traction in a Patient with Central Retinal Vein Occlusion: A Case Report

Fovea: 268,66
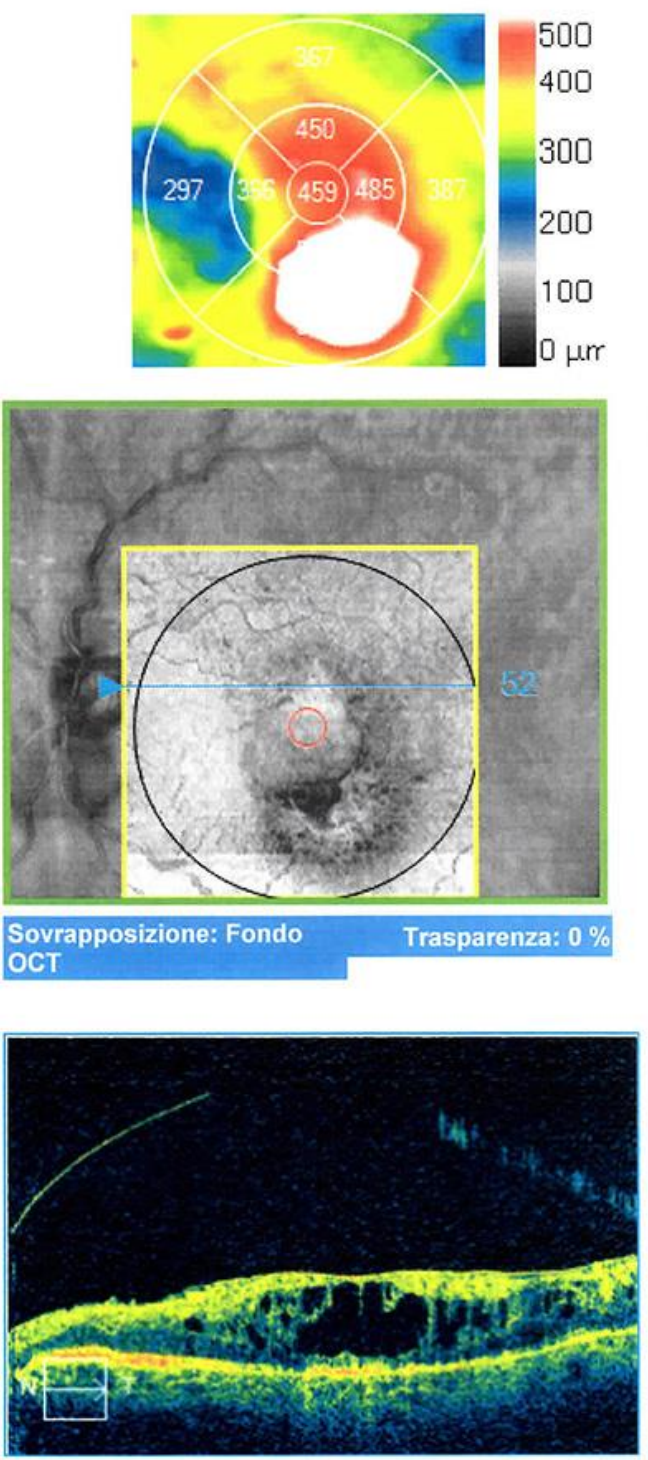

Fovea: 268,66
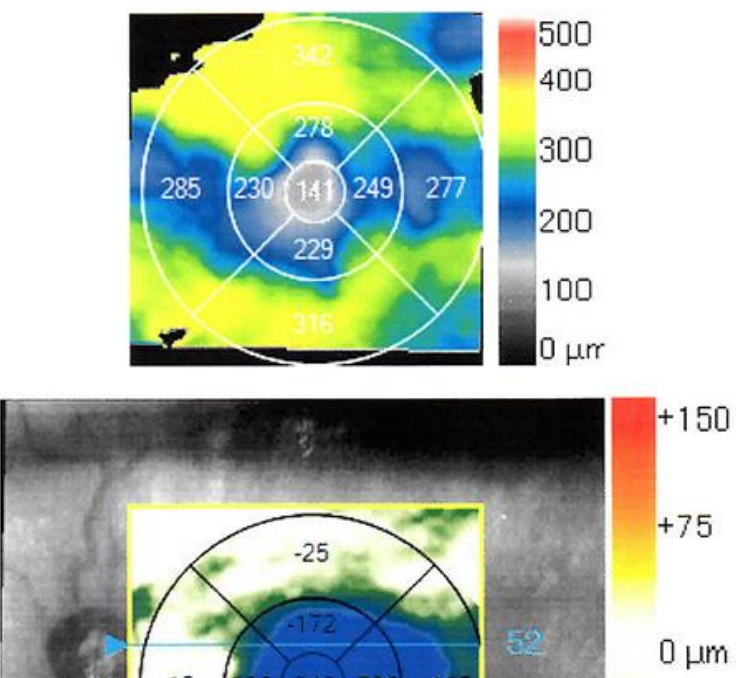

$0 \mu \mathrm{m}$

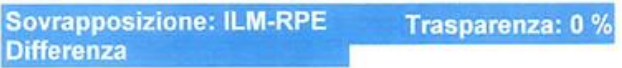

Scansione B estratta

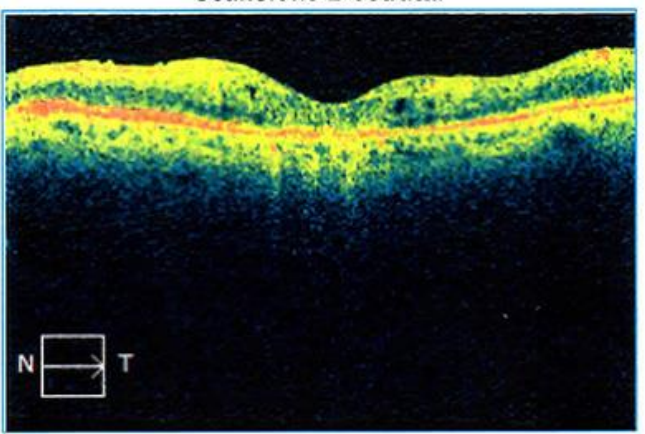

Fig. 3. Complete release of VMT with significant reduced foveal thickness $(141 \mu \mathrm{m})$, and reappearance of the foveal pit observed with SD-OCT 2 months after (August 2014) ocriplasmin intravitreal injection in the left eye. Nasal to the fovea, increased thickness persisted despite a remarkable reduction compared to the previous examination (May 2014). 


\section{Case Reports in Ophthalmology}

Case Rep Ophthalmol 2018;9:357-364 www.karger.com/cop

Rosati et al.: Ocriplasmin in the Treatment of Vitreomacular Traction in a Patient with Central Retinal Vein Occlusion: A Case Report
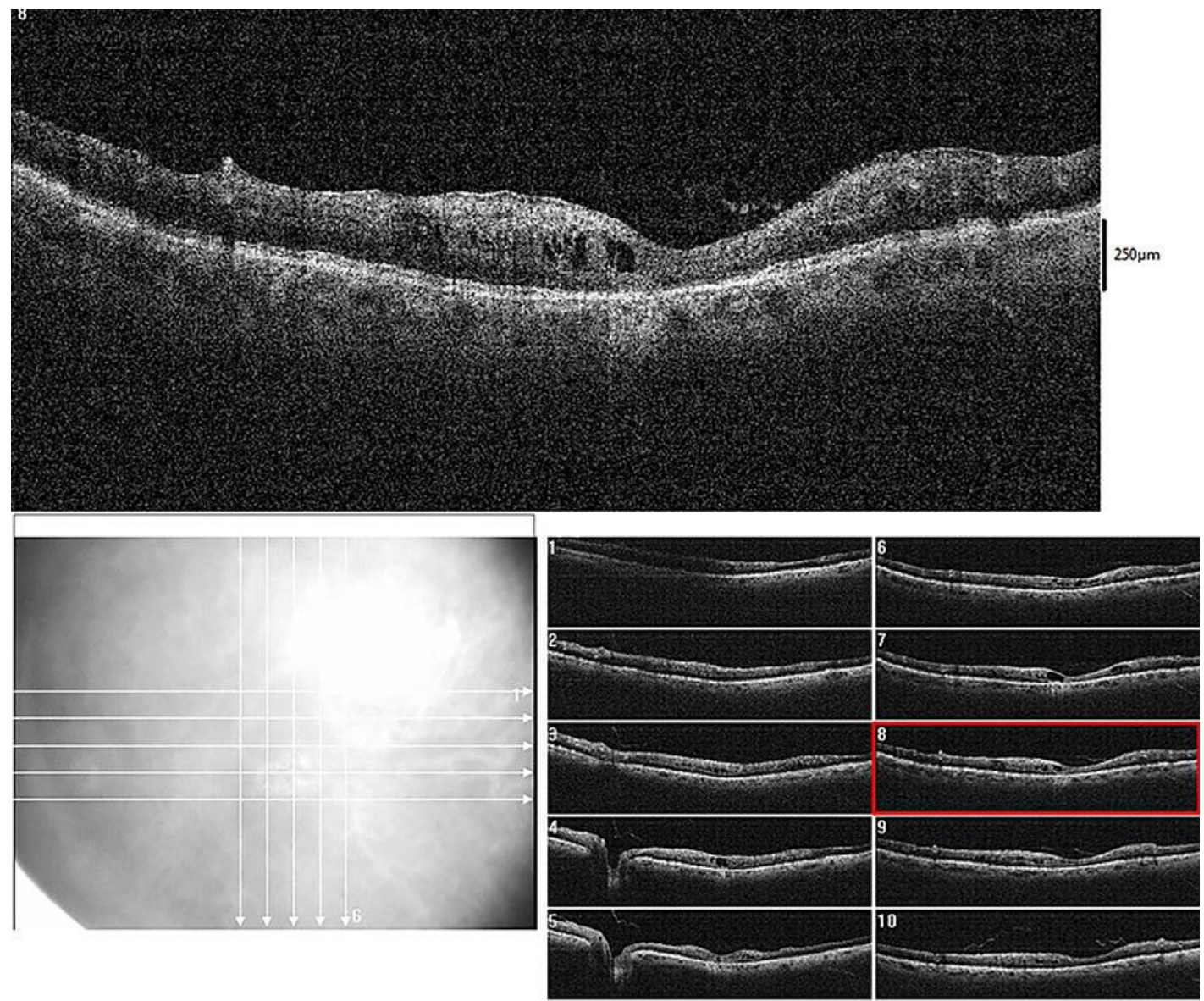

Fig. 4. SD-OCT performed in December 2014 demonstrated that the left eye remained stable. 